\title{
Between a rock and a workplace
}

\author{
Working spaces and cultures in the geosciences need to change in order to attract, safeguard and retain people \\ with disabilities.
}

\section{Anya Lawrence}

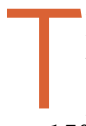
he one billion or so people living with disabilities represents one of the planet's largest minorities, making up $15 \%$ of the global population ${ }^{1}$. Physical, learning, sensory and unseen disabilities are present throughout societies regardless of race, ethnicity, age, religion, gender or sexual orientation. Students, staff and researchers who identify as disabled, however, remain sorely under-represented in academia, and the geosciences are no exception ${ }^{2}$.

Field-based disciplines like the geosciences present well-established challenges to those with disabilities. As such, over the past decade, increasing emphasis has been placed on making fieldwork more accessible and also on promoting officeor laboratory-based careers within the geosciences that do not involve a fieldwork component ${ }^{3}$. Yet, for disabled geoscientists, fieldwork isn't the whole story. The institutional workplace and its cultures can prove just as inaccessible and exclusionary as the most rugged and inhospitable environs.

\section{Exclusion from the outset}

When one has a disability, starting out in academic geoscience can feel like an impossible task. Workplaces often present a host of physical and sensory issues ranging from a lack of accessible bathrooms to laboratories with flickering lights to lecture halls that are unable to accommodate wheelchair users ${ }^{4}$.

While it's easy to assume that home-based working, which has proliferated during the COVID-19 pandemic, could augment all accessibility problems, this is not necessarily the case. Geoscientists often require access to specialist equipment and facilities to carry out their research; one cannot readily run rock powders through an XRF spectrometer, take CT scans of fossils or conduct thermogravimetric analyses of clay minerals at home.

It follows that many disabled geoscientists face a dilemma from the outset. Staying at home offers more flexibility in work patterns and control over the working environment, but substantially constrains the scope of one's research. In contrast, at the institutional workplace the research possibilities can be almost limitless but one has to endure being in an environment that often wasn't built with disability in mind a feat that requires great emotional labour and resilience on the disabled individual's part $^{5}$.

\section{Falling flat when 'fitting in'}

As well as the inaccessibility of the physical workplace, departmental attitudes can be a source of exclusion for disabled people. Outward indications of disabilities can draw negative responses; inability to verbalize or give eye contact may be perceived as disinterest or, worse, incompetence ${ }^{6}$, and similarly academic ability may be judged on the basis of physical appearance alone ${ }^{7}$.

Furthermore, workplace cultures aren't always inclusive. For example, the ubiquitous morning coffee break, often perceived to promote wellbeing, good conversation and networking amongst staff, can actually present an anxiety-provoking test of endurance to those with sensory or neurological disabilities rather than something to look forward to.

It is in such seemingly simple situations as these that disabled people must make the most difficult decisions. Trying to fit in with the normative departmental culture risks one's mental and physical health but bears the allure of potential social acceptance and inclusion. Likewise, playing it safe and staying away can lead to being typecast as the office recluse and forever met with fear, suspicion and pity (or combinations thereof) from one's able colleagues ${ }^{8}$.

\section{Ableist academic systems}

Many of the building blocks of academic systems are inherently ableist. The requirement to continuously produce research makes little allowance for those who need more time to process information ${ }^{9}$ or need to pace their work to manage chronic pain and fatigue. Pressure to disseminate research through seminars, conferences and public outreach talks (in both virtual and face-to-face formats) automatically excludes those who are non-verbal or verbally limited from making positive contributions.

It can thus be seen that the sustained demonstration of 'excellence' that modern academia incessantly demands ${ }^{10}$ is at odds with disabled people whose very appearance, communication style or work patterns defy notions of convention and normality.

\section{Gaining ground}

In recent years, especially during the pandemic, there has been much looking-inwards from the geoscience community regarding systemic barriers to inclusion for disabled people and other marginalized groups. However, while it's important to discuss problems pertaining to diversity, action is needed to gain ground in addressing these issues. The time has come for looking outwards, as so often solutions already exist; it's just knowing where to find them and how they can be recontextualised.

Embedding inclusion in the workplace is by no means a novel idea; numerous social enterprises and consultancy firms can offer bespoke guidance, assessments and coaching to help organizations, including multinational corporations like Microsoft and JPMorgan Chase and Co., to create and maintain working environments and practices that accommodate those with disabilities ${ }^{11}$. Therefore, while bold disability statements are a start, realizing these ambitions will depend on institutional leaders seeking and listening to the readily available advice from outside agencies and following the examples of best practice in other sectors.

Alongside institution-wide change, funding bodies and departmental managers specifically involved with the geosciences must also make visible commitments to disability-hiring initiatives ${ }^{12}$. More managerial positions need to be filled by disabled people, who have personal insight into the everyday realities of being 'other'. In this way, those with lived experience are actively involved in decision-making and can help create respectful cultures and welcoming spaces within departments where disabled scholars can thrive.

We, as geoscientists, can also learn much from our colleagues in the humanities and social sciences who are increasingly exploring collaborative research involving mixed groups of disabled and non-disabled academics, in which the strengths of 
different members are used to make up for the challenges experienced by other individuals within the group ${ }^{13}$.

Finally, in the geosciences, we are well-versed in time - after all, we have even managed to categorize the entire history of Earth into comprehensible intervals, each marked by key events. It is therefore essential that, starting today, we make meaningful changes so that the systemic exclusion of disabled people from the geosciences doesn't become the lasting legacy of the Anthropocene.
Anya Lawrence (iD $\square$

School of Geography, Earth and Environment, University of Birmingham, Birmingham, UK.

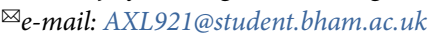

Published online: 10 June 2021

https://doi.org/10.1038/s41561-021-00775-4

\section{References}

1. Disability and Higher Education: Workforce Preparedness for Students with Disabilities (United Nations, 2020); https:// go.nature.com/3fnRXen

2. Carabajal, I. G., Marshall, A. M. \& Atchison, C. L. J. Geosci. Educ. 65, 531-541 (2017)

3. Stokes, A., Feig, A. D., Atchison, C. L. \& Gilley, B. Geosphere 15, 1809-1825 (2019).

4. De Picker, M. Disabil. Soc. 35, 163-167 (2020).
5. Inckle, K. Disabil. Soc. 33, 1372-1376 (2018).

6. Kingsbury, C. G., Sibert, E. C., Killingback, Z. \& Atchison, C. L. J. Geosci. Educ. 68, 302-310 (2020).

7. Brown, L. \& Boardman, F. Soc. Sci. Med. 72, 23-30 (2011).

8. Stone, D. L. \& Colella, A. Acad. Manag. Rev. 21, 352-401 (1996).

9. Olsen, J., Griffiths, M., Soorenian, A. \& Porter, R. Scand. J. Disabil. Res. 22, 265-274 (2020).

10. Moore, S., Neylon, C., Paul Eve, M., O’Donnell, D. P. \& Pattinson, D. Palgrave Commun. 3, 16105 (2017)

11. Annabi, H. et al. Autism @ Work Playbook: Finding Talent and Creating Meaningful Employment Opportunities For People With Autism (ACCESS-IT, The Information School, Univ. Washington, 2019)

12. Erickson, W. A., von Schrader, S., Bruyère, S. M., VanLooy, S. A. \& Matteson, D. S. Rehab. Res. Policy Educ. 28, 309-328 (2014).

13. Rosqvist, H. B. et al. Disabil. Soc. 34, 7-8 (2019).

Competing interests

The author declares no competing interests.

\section{Manage fire regimes, not fires}

Check for updates

Globally, land- and fire-management policies have counterproductively caused cascading ecosystem changes that exacerbate, rather than mitigate, wildfires. Given rapidly changing climate and land-use conditions that amplify wildfire risk, a policy shift to adaptive management of fire regimes is urgently needed.

\section{Mark A. Cochrane and David M. J. S. Bowman}

$\mathrm{T}$ he litany of catastrophic wildfires, causing death and destruction in anthropogenic landscapes, grows year after year: the United States in 2016, 2017, 2018 and 2020; Australia in 2009, 2019 and 2020; Chile in 2017; Portugal/Spain in 2017; Canada in 2016; and Indonesia in 2015 and 2019. Climate change intensified these fires, but changes in land and fire use compound issues by enhancing wildfire risk. From savannas to shrublands, wetlands to mountains, and boreal forests to rainforests, unforeseen fire problems develop wherever agricultural societies transform landscapes and disrupt indigenous fire-management practices. Societies respond to wildfire threats with various strategies such as fire bans, land and fuel management, and outright fire suppression. Yet rather than solving the problem, some of these approaches make the original fire issue even more problematic. This is because settler societies fail to grasp what pre-agricultural peoples understood: fire and vegetation are dynamically linked - changing either one alters the other ${ }^{1}$. Human land uses set in motion processes that often culminate in unmanageable wildfires. Land-management policies and practices must recognize that wildfires are a persistent condition of flammable landscapes requiring adaptive management ${ }^{2}-$ not a 'solvable' problem.
Wildfires are not external ecological disturbances. They are an emergent property of climate, terrain, biological properties and human activities within a landscape. These factors shape fire regimes - the typical range of fire frequency, intensity, type (ground, surface or crown), severity, seasonality, spatial scale and internal heterogeneity. Not every terrestrial ecosystem is fire-dependent, but all can burn under the right conditions. Where fire is prevalent, evolutionary adaptions within plant species have proceeded to varying degrees. Shifting climates, altered land cover, and human land-use changes combine to establish new relationships between an ecosystem and wildfire behaviour.

\section{Human fire and land use}

Landscapes were shaped by human fire and land uses long before anthropogenic climate change ${ }^{3,4}$. Prehistoric humans skilfully used fire for domestic chores, such as cooking, and also for broader landscape applications, such as hunting and land-cover modification. Setting many small low-intensity fires created 'pyrodiverse' habitat mosaics that support different plant and animal species ${ }^{5}$.

The advent of agriculture disrupted indigenous fire management and fundamentally changed how human fire and land uses were linked. A typical arc of land use and fire continues to play out globally in forested landscapes. Poorly managed timber operations leave behind widespread forests littered with tons of woody fuels. The expansion of human settlements clears these woody fuels with fire when converting forest to agricultural land. Pervasive use of fires for land management within an increasingly flammable landscape makes catastrophic wildfires inevitable once extreme fire weather conditions occur. The threats to fire-sensitive permanent settlements, agricultural land uses and infrastructure promote a cultural aversion to wildland fire that drives policies and practices that further disrupt fire regimes through, often aggressive, fire suppression efforts.

Human activities have shaped the modern fire regimes for most ecosystems. With the exception of lightning in wilderness areas, human activities cause the vast majority of the world's wildfires. Ignitions concentrate in frontier rural landscapes, rising together with population density, until urbanization processes take over and suppress fire numbers ${ }^{6}$. Landscape fragmentation by roads and fields, and fire control efforts, restrict the extent of the vast majority of fires. As a result, woodlands adjacent to urban areas burn less frequently, favouring woody growth, accumulation of potential fuels and invasion by fire-sensitive species. Denser canopies shade out shrubs and grasses, further inhibiting fire spread. 